\title{
Journal of Biomolecular Structure and Dynamics
}

\section{In silico inhibition of SGTP4 as a therapeutic target for the treatment of schistosomiasis}

Tayo A. Adekiya, Raphael Taiwo Aruleba , Ashwil Klein \& Adewale O. Fadaka

To cite this article: Tayo A. Adekiya , Raphael Taiwo Aruleba , Ashwil Klein \& Adewale O. Fadaka (2020): In silico inhibition of SGTP4 as a therapeutic target for the treatment of schistosomiasis, Journal of Biomolecular Structure and Dynamics, DOI: 10.1080/07391102.2020.1850363

To link to this article: https://doi.org/10.1080/07391102.2020.1850363

曲 Published online: 23 Nov 2020.

Submit your article to this journal

山 Article views: 29

Q View related articles $\widetilde{ }$

View Crossmark data $־$ 


\title{
In silico inhibition of SGTP4 as a therapeutic target for the treatment of schistosomiasis
}

\author{
Tayo A. Adekiya ${ }^{a}$ D, Raphael Taiwo Aruleba ${ }^{b}$ (D) Ashwil Klein ${ }^{c}(\mathbb{D})$ and Adewale O. Fadaka ${ }^{d}$ \\ ${ }^{a}$ Wits Advanced Drug Delivery Platform Research Unit, Department of Pharmacy and Pharmacology, School of Therapeutic Science, Faculty \\ of Health Sciences, University of the Witwatersrand, Johannesburg, South Africa; ${ }^{b}$ Department of Molecular and Cell Biology, Faculty of \\ Science, University of Cape Town, Cape Town, South Africa; 'Department of Biotechnology, Faculty of Natural Sciences, University of the \\ Western Cape, Bellville, South Africa; ${ }^{\mathrm{d} D e p a r t m e n t}$ of Science and Innovation/Mintek Nanotechnology Innovation Centre, Biolabels Node, \\ Department of Biotechnology, Faculty of Natural Sciences, University of the Western Cape, Bellville, South Africa
}

Communicated by Freddie R. Salsbury

\section{ABSTRACT}

Schistosomiasis is an infectious tropical disease caused by parasitic flatworm of the genus Schistosoma. This debilitating disease chronically infects about 200 million people globally and management relies on chemotherapy. Unfortunately, the solely available schistosomicide (praziquantel) against all forms of adult schistosmes has been faced with numerous drawbacks. Thus, there is an urgent need to design and develop a new regimen for schistosomiasis. In light of this, the current study focuses on inhibiting the schistosome glucose transporter 4 (SGTP4) as a therapeutic candidate for schistosomiasis. Several studies have revealed that Schistosoma parasites require an adequate amount of energy/glucose to survive. We modelled the 3D structure and subsequently used the homology model for docking with praziquantel (PZQ), Licochalcone A, Licarin and Harmonine. The docked complexes were subjected to molecular dynamics using Desmond system of Schrodinger software. Furthermore, the pharmacokinetic parameters of the ligands were investigated using the QikProp tool in the Schrodinger-2019-4 software suite. After performing all the computational analysis, our findings reveal that all four ligands were able to inhibit SGTP4 effectively through the higher glide $G$ score (dock score) of $-5.8(-5.8),-6.5(-6.4),-7.3(-7.3)$ and $-4.9(-4.9)$ in $\mathrm{kcal} / \mathrm{mol}$ for praziquantel, licochalcone $A$, licarin and harmonine respectively against the protein. The molecular simulation further confirmed that the stability of the complexes formed between the ligands and protein is excellent. More so, all the ligands fulfilled oral drugability of both the Lipinski's rule of five and Veber's rules.

The findings in this present study provide new useful insights for the design of drugs which can serve as an alternative to praziquantel in the treatment of schistosomiasis through the inhibition of SGTP4.
ARTICLE HISTORY

Received 5 October 2020

Accepted 6 November 2020

KEYWORDS

SGTP4; schistosomiasis; praziquantel; antischistosomal; computational biology; drug

\section{Introduction}

For many decades, schistosomiasis continues to be on a swift and rapid increase globally, claiming thousands of lives per annum and leaving over 800 million persons at risk of infection (WHO, 2020). Currently, it's the second most prevalent among the groups of neglected tropical diseases (NTDs), which are acute and chronic infectious diseases that have been forsaken for many years (Adekiya et al., 2020a). Schistosomiasis is caused mainly by Schistosoma mansoni (S. mansoni), S. haematobium and S. japonicum. It has been reported that the disease poses a great negative impact on agricultural produces and severe effects on pregnant women and the development and life of school children in affected regions (Adekiya et al., 2017). This disease has been reported to be under the group A causing agents of several cancer types such as; liver, bladder, intestine among others, the coinfection has also been reported with other chronic ailments such as HIV-AIDS. Over the years, PZQ remains the only drug of choice and the best therapeutic agent due to its effectiveness against all forms of schistosomes (Aruleba et al., 2019). This drug is very cheap, easily accessible and possesses lesser side effects which are tolerable in patients of all ages. Sadly, some shortcoming has been reported on PZQ, some of which include; rapid metabolism, which reduces the bioavailability of the drug in the circulation, drug resistance in some parts of the world, ineffectiveness against the younger forms of the schistosomes worm and unable to prevent the reinfection of schistosomiasis (Aruleba et al., 2019) hence, the need for an alternative drug molecule.

In recent times, the use of a computational approach has been a great technique in medicinal chemistry for the screening and evaluation of novel drug molecular compounds capable of interacting with specific molecular receptors. Thus, the interaction of some selected potential antischistosomal compounds (Licochalcone A, Licarin, Harmonine and praziquantel as control) was investigated on one of the prominent targeted protein found in the tegument of 
Schistosoma species. Recently, Adekiya et al. (2020b) highlighted several potential druggable and vaccine proteins targets and molecular receptors found on the surface of the schistosome tegument such as; schistosome glucose transporter 1 (SGTP1) and 4 (SGTP4) which are found in all types of schistosomes (Skelly et al., 1994, 1998), acetylcholinesterase (AChE) and a nicotinic type of acetylcholine receptor (nAChR) which are primarily located on the surface of male schistosomes (MacDonald et al., 2014; Mansour \& Mansour, 2002). Other targeted protein found on the surface of the tegument is dynein (S. mansoni) (Mansour \& Mansour, 2002). For the purpose of this study, SGTP4 was the target protein because, SGTP4 transporter protein is distributed evenly on the ventral and dorsal surfaces of male and female schistosome teguments, which has a unique double lipid bilayer structure (Mansour \& Mansour, 2002). Several studies have revealed that Schistosoma parasites require an adequate amount of energy/glucose to survive (Krautz-Peterson et al., 2010; Skelly \& Shoemaker, 1996; Skelly et al., 1994, 1998). This glucose/energy is first taken up by the tegument of the schistosomes and not by the caecum of the intestine, and its uptake inside the tegument of the schistosomes are facilitated by the help of the schistosome glucose transporters, which are found in schistosome tegument (Krautz-Peterson et al., 2010). The distinctive location of the SGTP4 on the membrane tegument indicates that the protein happens to be the transporter that facilitates the movement of glucose from the host bloodstream into the parasite tegument (Skelly \& Shoemaker, 2001). Furthermore, SGTP4 controls the developmental stages and appear during the transformation of free-living cercariae into schistosomula during growth where it fulfils the needs of the parasites for the high uptake of glucose once they enter the human-host (schistosomula stage) and throughout adulthood (Skelly \& Shoemaker, 1996). Thus, this study evaluates the several aspects behind the anti-schistosomal activity of Licochalcone A, Licarin, Harmonine against SGTP4 protein receptor. Moreover, praziquantel, a standard and the only drug of choice for schistosomiasis treatment was docked against SGTP4 protein receptor for comparison.

\section{Materials AND methods}

\subsection{Homology modelling and preparation of SGTP4 as a drug target}

The SGTP4 sequence was retrieved in FASTA format from the Uniprot database with accession no Q26581_SCHMA. Thereafter, the suitable template for homology modeling was identified by performing a blast search on protein data bank (PDB) since the crystal structure of the SGTP4 is not available on the PDB. The solute carrier family 2, facilitated glucose transporter member 3 (4ZW9: A) was identified and the sequence was used to model the 3D structure of SGTP4 using the Schrodinger Suite. The target and the template were aligned and all other PRIME parameters were adjusted accordingly. Loops were refined and subsequently, the refined homology model was utilized for downstream steps. The structure was opened using the protein preparation wizard in Maestro and using a method described by Fadaka et al. (2019) we optimized PropKa, generated the het states using EPIK, alleviated steric clashes by force field: OPLS_2005 and PRIME was used to fix missing atoms. Finally, the stability and overall quality of the homology modelled structure of the SGTP4 was assessed using RAMPAGE (Laskowski et al., 1996).

\subsection{Ligands selection and preparation as inhibitors of SGTP4}

In this study, we used five ligands that have been reported in literature as schistosomicide or potential schistosomicide. Amongst the five ligands, praziquantel (pubchem ID-4891) was used as our control because it is the only available chemotherapy active against all forms of adult Schistosoma spp. and other ligands retrieved from pubchem (Kim et al., 2019) are licochalcone A (pubchem ID-5318998), licarin (pubchem ID-73548) and harmonine (pubchem ID-14825482). Of course, removing salt and additional hydrogen atoms are key to obtaining good and reliable protein-ligand docked complex. Hence, all ligands in their 3-D structure format were subjected to ligand preparation (LigPrep) of the Schrodinger Maestro suite. They were optimized via Epik 25 in pH between $7 \pm 2$ and ring conformation, desalting, sterochemistries and tautomer generation were selected to get at least 32 conformations for each ligand.

\subsection{Admet/tox screening}

In the drug discovery platform, identification of new molecules with sort after pharmacokinetic and ADME/Tox profile is a challenge. Hence, the prediction of these properties using computational techniques is considered a vital step in the screening for a new tolerable compound with little or no effects on the body (Akabli et al., 2019). The pharmacokinetic parameters of the ligands (praziquantel, licochalcone A, Licarin, Harmonine) were investigated using the QikProp tool in the Schrodinger-2019-4 software suite. Subsequently, we forecasted the toxicological properties of the selected ligands using admetSAR online device (http://Immd.ecust.edu.cn/ admetsar1/predict/) to ascertain if they fulfilled the Lipinski's and Veber rules (Lipinski et al., 2001; Veber et al., 2002). And here, we predicted the acute oral toxicity, carcinogenic properties, ames toxicity and rodent acute toxicity.

\subsection{Generation of grid and molecular docking of SGTP4 against the ligands}

The generation of the receptor grids is vital as it identifies the binding area between a ligand and the receptor. The receptor grid generation application of the maestro version 12.2 was used to define the active binding site in terms of coordinates $x, y$ and $z$ by indicating the amino acid residues which have been reported previously for the protein. Here, the receptor grid box resolution was centered at coordinates -20.57 (x-axis), 18.10 (y-axis), and -26.99 (z-axis). After successfully generating the grid, we performed molecular 


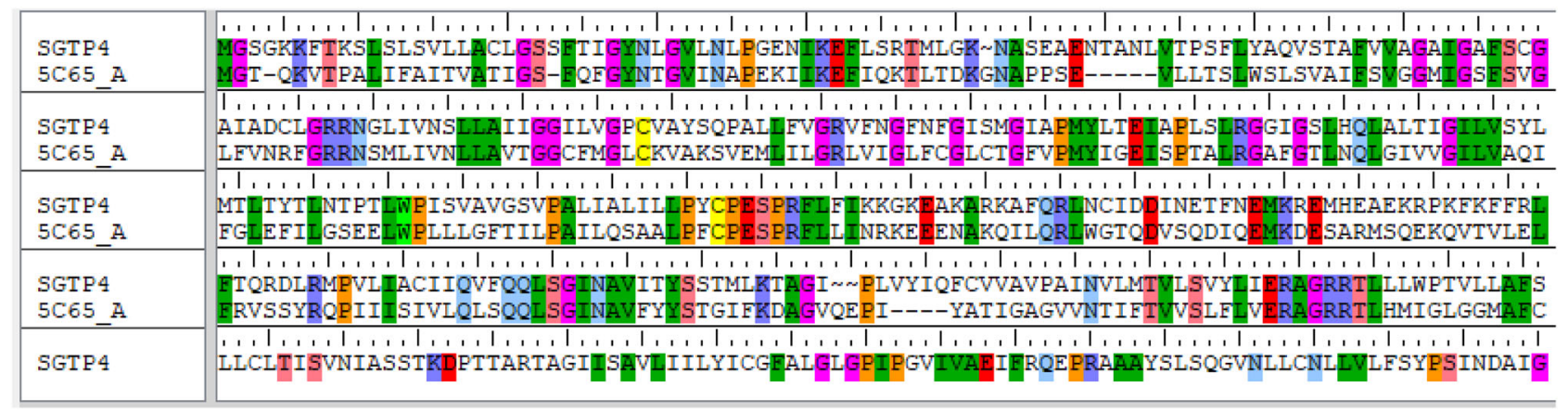

Figure 1. Alignment step of SGTP4 sequence with template (4zw9: A) Coloured by default.

docking on the prepared receptor against each of the four ligands using the Glide tool in Maestro. All ligands were first docked unto the active site of SGTP4 using standard precision (SP) and followed by extra precision (XP) to decrease the intermediate conformations. The interaction diagram of the ligands with amino acid residues at the active site of the SGTP4 was visualized using the ligand interaction tool.

\subsection{Prime molecular mechanics, the generalized born solvent accessibility (MM-GBSA) calculation}

The binding free energies $\left(\Delta G_{\text {bind }}\right)$ of the docked complexes were calculated using the MM-GBSA method, implemented in Schrodinger suite software. This post docking tool is crucial because it aids the prediction of relative free binding energy with an acceptable level of accuracy and provides the correct ranking of ligands binding (Lyne et al., 2006). Here, the PRIME module embedded in Maestro was employed to ascertain the free binding energies of the docked complexes obtained from the XP docking step. Briefly, the energies of the various complexes were calculated using the OPLS_2005 force field in relation to GBSA continuum model.

\subsection{Molecular dynamics simulation}

All the docked complexes were subjected to molecular dynamics (MD) using Desmond system builder panel in Schrodinger with OPLS 2005 force field and each complex was restricted with a predefined TIP3P water molecule in orthorhombic box (Jorgensen et al., 1983). Subsequently, the box was reduced and the ligand-receptor complex was neutralized by adding sodium and chloride ions. The system was equilibrated at constant temperature (300 Kelvin) and standard atmospheric pressure (1.01325 bar) using methods by Hoover thermostat and Martyna-Tobias-Klein barostat (Essmann et al., 1995; Hoover, 1985). The long-range electrostatic interactions were determined by Particle-Mesh-Ewald method (Hoover, 1985). The RMSD plots for backbone atoms were analyzed to identify the relative stability of the ligand in the binding pocket of the receptor.

\section{Results and discussion}

The urgent need for a new schistosomicide cannot be overemphasized due to challenges faced by the sole available PZQ. Designing a structure-based drug with high efficacy could elude these problems faced by the existing drug. Indeed, conventional methodologies employed in the identification and development of inhibitors are capital and timeconsuming, hence, computational techniques have been used and gained importance in recent years (Adekiya et al., 2017; Aruleba et al., 2018, 2020; Fadaka et al., 2020; Ojo et al., 2019; Oyinloye et al., 2019).

\subsection{Homology modelling}

Due to the unavailability of the resolved 3-D structure of the SGTP4 protein in PDB, we generated one through homology modelling using BLAST $P$ against existing structures in the PDB. The crystal structure of 4ZW9: A was identified and selected with a sequence similarity of $37.83 \%$ with the SGTP4 protein sequence (Figure 1). And it has been documented that a sequence similarity greater than $25 \%$ is needed to perform homology modelling (Yadav \& Khandelwal, 2019). Subsequently, modelling was performed using the PRIME module of the Schrodinger suite and depicted in Figure $2 \mathrm{~A}$ is the modelled structure of the SGTP4 protein and the superimposed of the template. The homology structure was subjected to refinement by the Protein Prep wizard in Maestro (Figure 2b). In essence, this is done to stabilize the protein by the addition of hydrogen bond, optimize the structure and minimize the energy. Furthermore, the quality of the modelled SGTP4 was validated using PROCHECK, a program for structure verification that relies on principle on Ramachandran plot (Aruleba et al., 2018). The results (Figure 2c) reveal that the structure has $93.2 \%$ amino acid residues in the most favoured regions, a total of $6.6 \%$ residues in both additional and generously allowed regions and $0.2 \%$ residues in the disallowed regions. This signifies that the built model is of a high quality due to the excellent distribution of amino acids in the allowed regions.

\subsection{Docking}

The binding affinity between all the potential anti-schistosomal molecules and SGTP4 protein was evaluated by in silico molecular docking analysis. The three-dimensional structure 

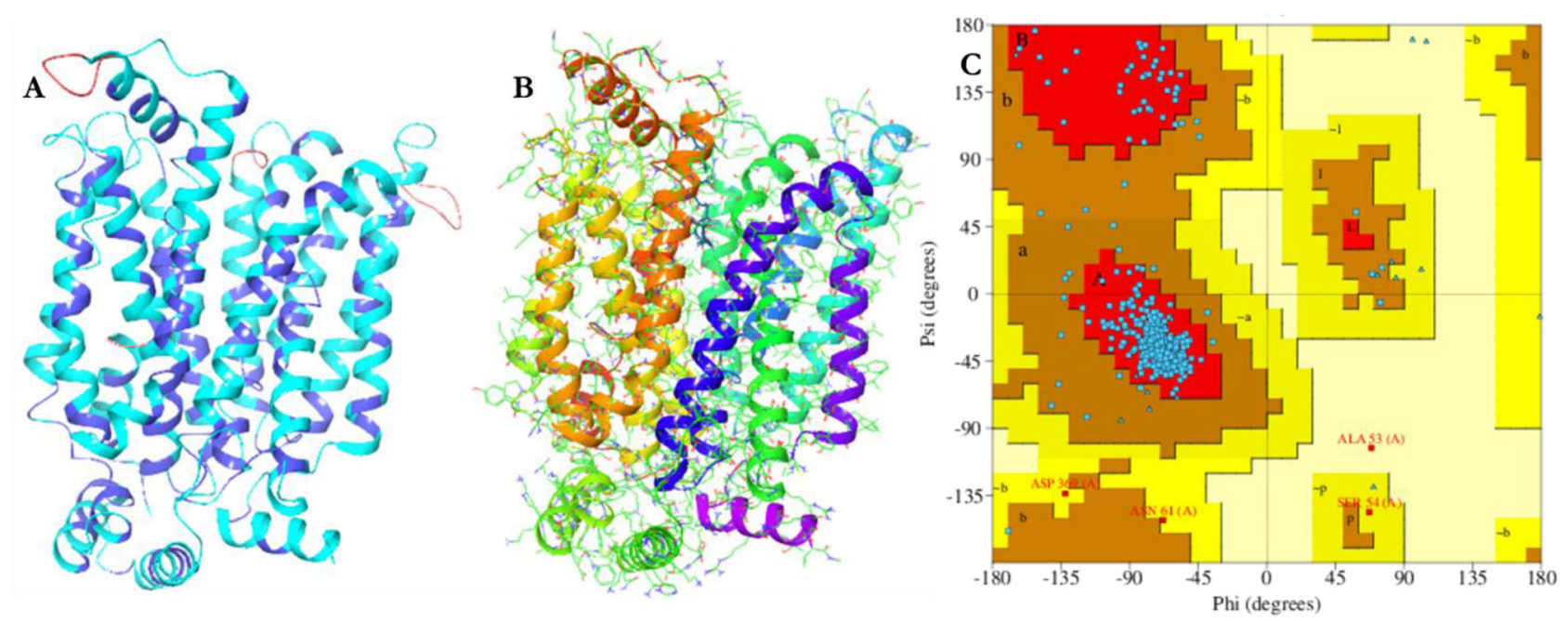

Figure 2. Modeled structure of SGTP4 using 4ZW9: A template. Modeled (A), prepared protein (B), and quality check (C).
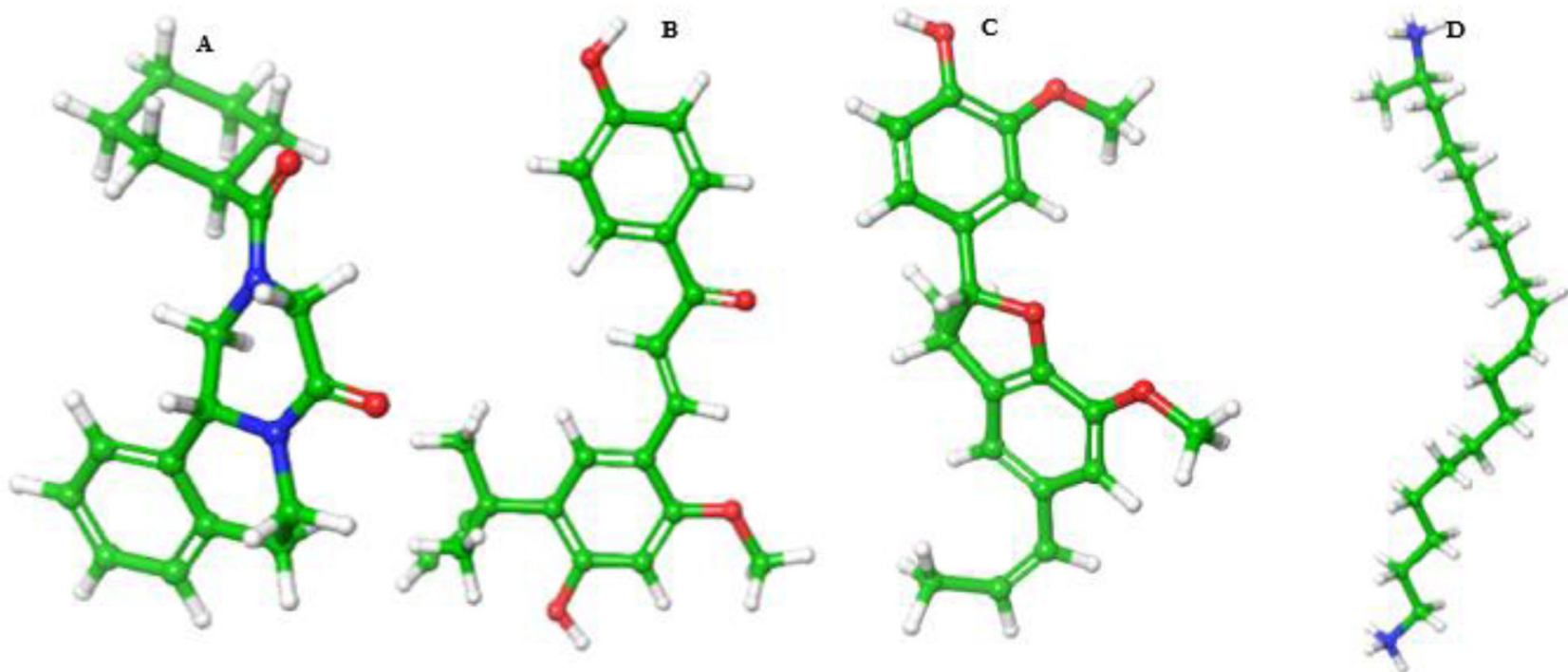

Figure 3. Prepared 3D structures of the ligands. Praziquantel (control)-ID:4891 (A), Licochalcone A-ID:5318998 (B), Licarin-ID:73548 (C), Harmonine-ID:14825482 (D).

of all the druggable molecules was retrieved in 3D sketcher and imported into the Schrodinger Maestro suite workplace for preparation and optimization (Figure 3a-d), at least, 32 conformers for each ligand was generated. Subsequently, the ligands were subjected to docking with the active site of SGTP4, using SP and thereafter, XP in order to decrease the intermediate conformations. The $2 \mathrm{D}$ docking poses of all the best ligand conformers interaction with the active site of SGTP4 shown in Figure 4, were visualized with the help of ligand interaction tool. Here, results were described in terms of Glide score, Dock score, free binding energy and number of $\mathrm{H}$-bonds. Glide score showed that all the five ligands were energetically favorable in a similar trend (Table 1). However, praziquantel (a standard drug for schistosomiasis) have a glide binding score of $-5.8 \mathrm{Kcal} / \mathrm{mol}$ against SGTP4 but, do not generate hydrogen bond interaction with any amino acids of the protein receptor. Licochalcone $A$ isolated from Glycyrrhiza inflate increased reactive oxygen species in adult S. mansoni which acted on the tegument, mitochondrial and cellular membrane of the worm thereby inducing death of the parasite (Souza et al., 2017). In view of licochalcone A anti-schistosomal potentials, we docked it against SGTP4 and it showed a better binding score of $-6.5 \mathrm{Kcal} / \mathrm{mol}$ when compared to the control; praziquantel, with an essential interaction with Asn 292, which is important for the inhibition of SGTP4. More so, licarin A a neolignan isolated from different plant species was utilized here based on reported activity against S. mansoni (Meleti et al., 2020; Pereira et al., 2011).

The docking of licarin against SGTP4 showed good binding interaction of $-7.3 \mathrm{Kcal} / \mathrm{mol}$, but unfortunately, the ligand does not display any hydrogen bond interaction with any essential amino acids present in SGTP4 protein, which may be of importance for the inhibition or regulation of the protein. It was further revealed in our docking study that harmonine showed the least binding score of $-4.9 \mathrm{Kcal} / \mathrm{mol}$, but interestingly, displayed an important interaction with Glu 38, Asn 34 and Thr 296 of the protein. We selected harmonine based on results reported by Kellershohn and co-workers (Kellershohn et al., 2019) that it caused physiological, 


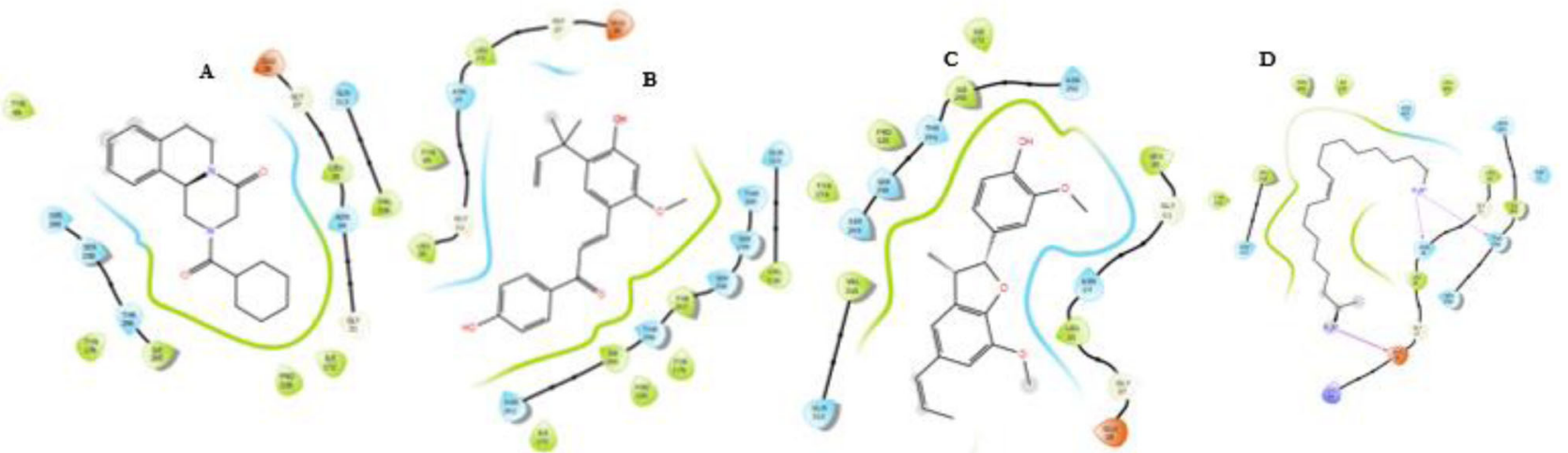

Figure 4. 2D docking interactions. Polar, hydrophobic and charged interacting residues. Praziquantel (A), Licochalcone A (B), Licarin (C), Harmonine (D).

Table 1. Docking results of the ligands and the energy generated in the active sites of the receptors.

\begin{tabular}{lcccc}
\hline Receptor & Glide Gscore & Dock score & $\Delta \mathrm{G}_{\text {bind }}(\mathrm{kcal} / \mathrm{mol})$ & (No) of H-bonds within 2.5 \\
\hline Praziquantel & -5.8 & -5.8 & -64.39 & $/$ \\
Licochalcone A & -6.5 & -6.4 & -62.74 & (1) ASN292 \\
Licarin & -7.3 & -7.3 & -69.40 & $/$ \\
Harmonine & -4.9 & -4.9 & -85.31 & (3) GLU38 ASN34 THR296 \\
\hline
\end{tabular}

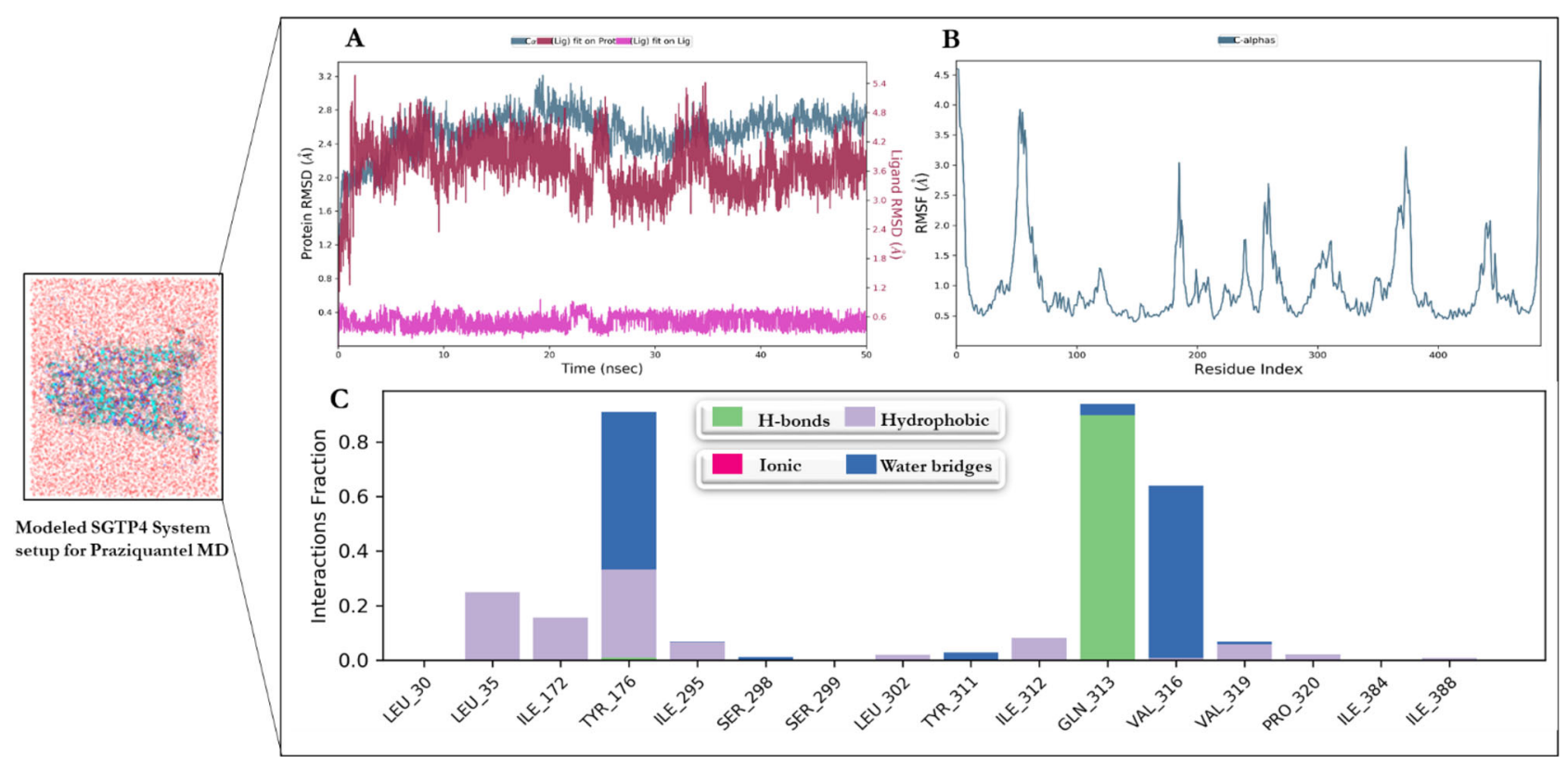

Figure 5. MD simulations: (A) Root mean standard deviation(RMSD) of Praziquantel with modeled SGTP4 complex as a function of 50 nanoseconds simulation time, (B) Root mean square fluctuation (RMSF) values of complex SGTP4 residues with Praziquantel, and (C) The SGTP4- Praziquantel contacts.

cellular and molecular changes like gut dilation, tegumental damage, impaired fecundity and reduced motility on S. mansoni. Thereafter, the MM-GBSA free energy calculations were used to evaluate the free binding energies of the ligands inside the SGTP4 active site. It was revealed that licarin $(-69.40 \mathrm{kcal} / \mathrm{mol})$ and harmonine $(-85.31 \mathrm{kcal} / \mathrm{mol})$ had a better MM-GBSA score than the PZQ $(-64.39 \mathrm{kcal} / \mathrm{mol})$. Nevertheless, licochalcone $A$ had a relatively lower MM-GBSA value of $-62.74 \mathrm{kcal} / \mathrm{mol}$ than PZQ.

\subsection{Analysis of molecular dynamics simulations}

MD simulations were used to calculate the docking results using the Desmond package in Schrodinger with OPLS
2005 force field and each complex was restricted with a predefined TIP3P water molecule in the orthorhombic box. The behaviour of the atomic positions of all the molecular compound interaction with the SGTP4 protein from the root mean square deviation (rmsd) (Figures 5a-8a) were studied and it was shown that there is no large variation in the binding simulation of compounds to the receptor. Thus, these results are in accordance with the docking results which showed that all the compounds depicted good glide binding score with the SGTP4 protein apart from licochalcone $A$ and licarin which showed slightly noticeable different atomistic behaviour (Figures 6a and 7a). However, the binding stability of all the compounds to the SGTP4 protein receptor could be depicted by the smaller variations of the rmsd. As shown in the RMSF results in Figure $5 b-8 b$, there 


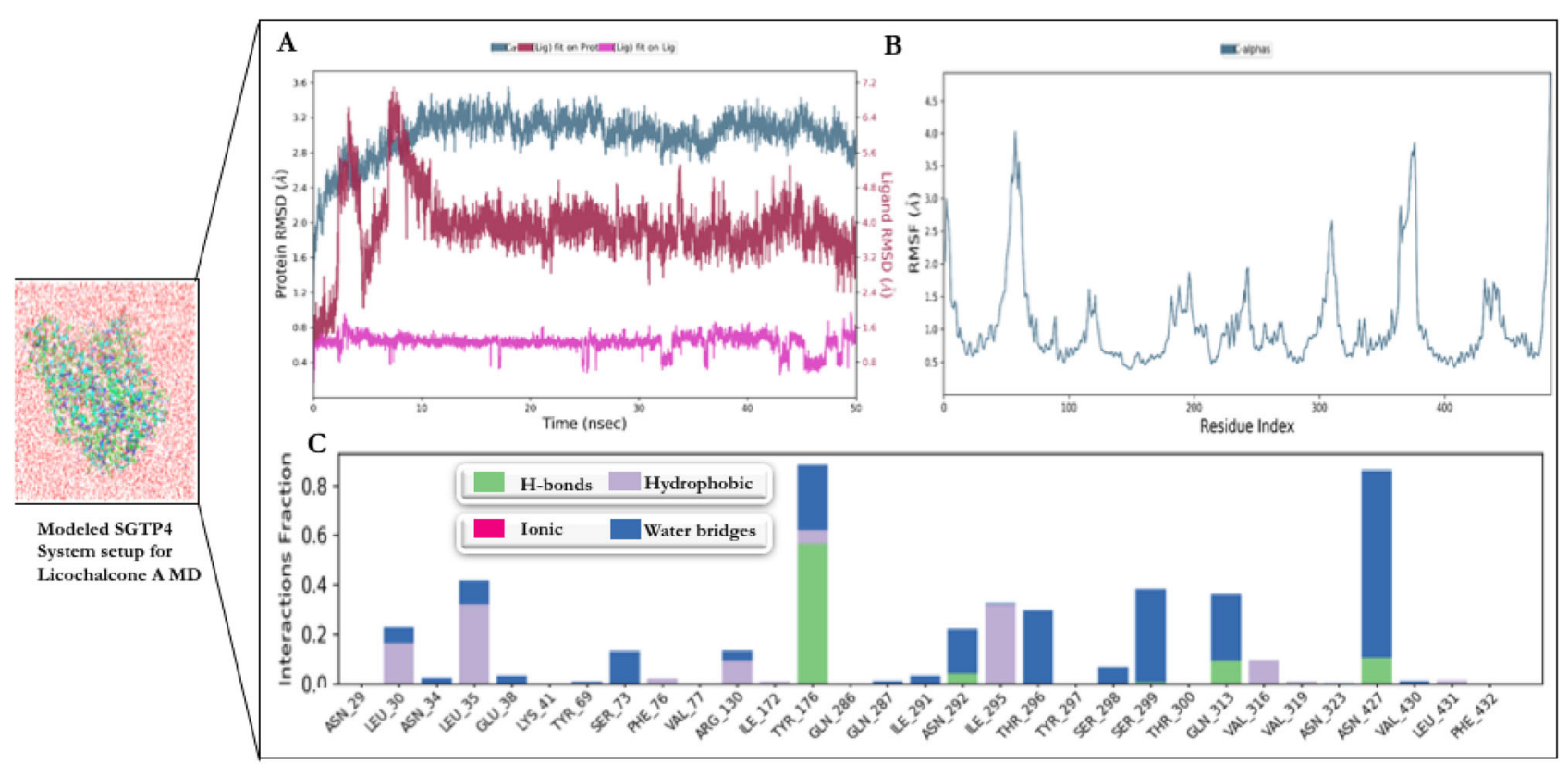

Figure 6. MD simulations: (A) Root mean standard deviation (RMSD) of Licochalcone A with modeled SGTP4 complex as a function of 50 nanoseconds simulation time, (B) Root mean square fluctuation (RMSF) values of complex SGTP4 residues with Licochalcone A, and (C) The SGTP4-Licochalcone A contacts.

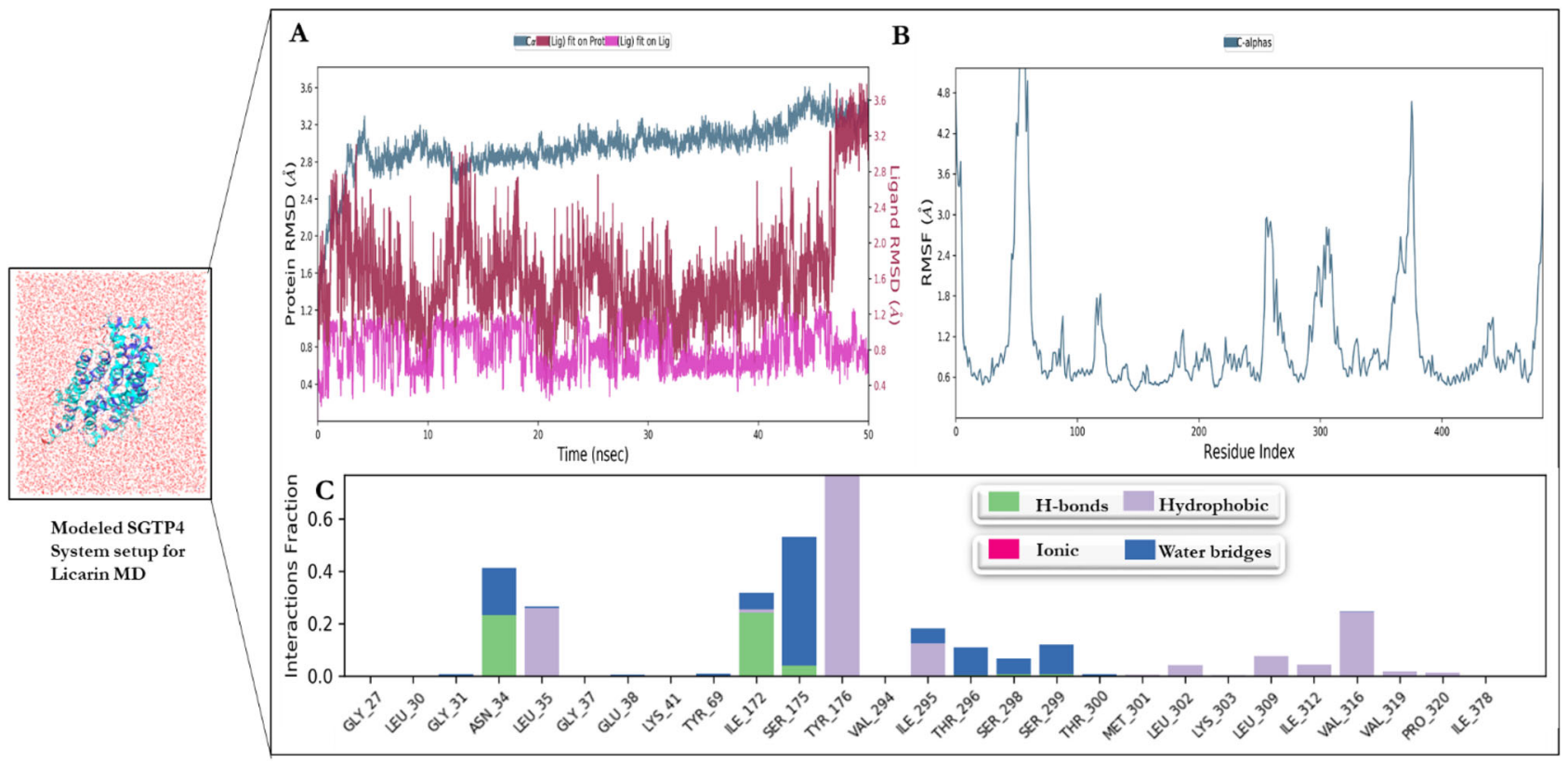

Figure 7. MD simulations: (A) Root mean standard deviation(RMSD) of Licarin with modeled SGTP4 complex as a function of 50 nanoseconds simulation time, (B) Root mean square fluctuation (RMSF) values of complex SGTP4 residues with Licarin, and (C) The SGTP4- Licarin contacts.

was deviation of different residues of SGTP4 when compared to the chosen reference molecule. Hence, both the RMSF and RMSD ligands showed consistent behaviour throughout the simulation period, this could further infer stability of the complexes.

The location of the binding or the interaction of the molecular compounds and SGTP4 was also evaluated by counterpart MD modelling. Interestingly, the MD results showed that all the compound interaction with the SGTP4 protein has a water bridge, hydrophobic, hydrogen interaction. Praziquantel; the control has contact hydrogen bond interaction with SGTP4 at residue GLN 313, while the drug formed water-bridge with the protein at residues TYR 176 and VAL 316. Praziquantel, the control drug has hydrophobic interaction at protein residues LEU 35, ILE 172, TYR 176, ILE 295 ILE 312 and VAL (Figure 5c), it was discovered that there are lesser contact interactions between praziquantel and SGTP4 when compared with all the potential anti-schistosomal compounds evaluated in this study. As shown in Figure $6 c$, we discovered a hydrophobic interaction between the licochalcone A and SGTP4 in protein residues LEU 30, LEU 35, ARG 130, ILE 295 and VAL 316. Hydrogen bonding was observed in TYR 176, GLN 313, ASN 427 and ASN 292, which was also seen in the docking results. There was significant 


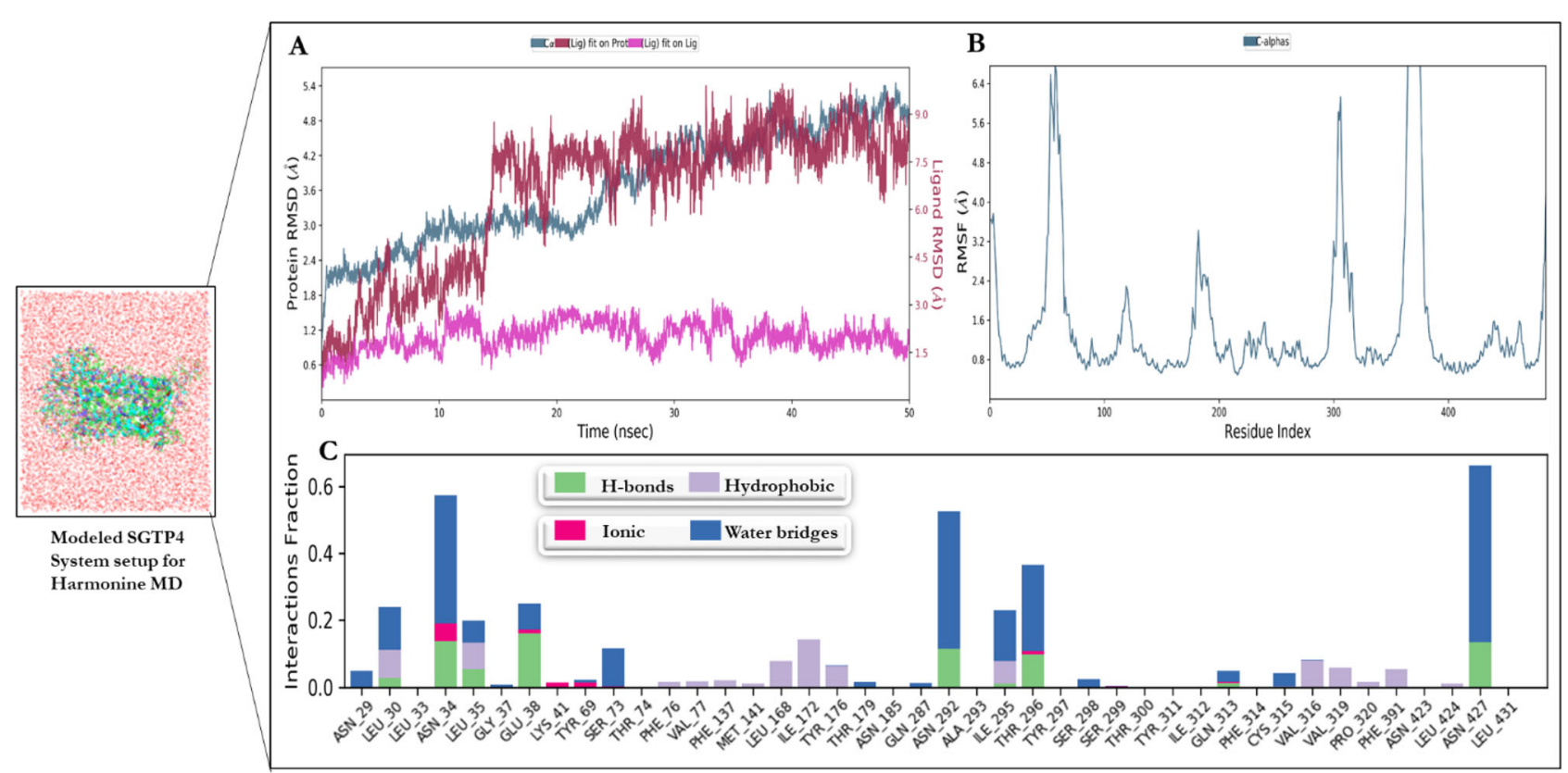

Figure 8. MD simulations: (A) Root mean standard deviation(RMSD) of Harmonine with modeled SGTP4 complex as a function of 50 nanoseconds simulation time, (B) Root mean square fluctuation (RMSF) values of complex SGTP4 residues with Harmonine, and (C) The SGTP4-Harmonine contacts.

Table 2. Pharmacological properties of the ligands.

\begin{tabular}{lcccccccc}
\hline & \multicolumn{4}{c}{ Lipinski rules } & & & \multicolumn{2}{c}{ Veber rules } \\
\cline { 2 - 5 } \cline { 8 - 9 } Compound & MW & HBA & HBD & Log $\mathrm{p}$ & Violations & nRB & TPSA \\
\hline ROF cut-off & $<500$ & $<10$ & $<5$ & $\leq 5$ & $\leq 1$ & $\leq 10$ & $\leq 140$ \\
Praziquantel & 312.41 & 2 & 0 & 2.40 & 0 & 2 & $40.62 \AA^{2}$ \\
Licochalcone A & 338.40 & 4 & 2 & 3.98 & 0 & 6 & $66.76 \AA^{2}$ \\
Licarin & 326.39 & 4 & 1 & 3.88 & 0 & 4 & $47.92 \AA^{2}$ \\
Harmonine & 282.51 & 2 & 2 & 4.72 & 0 & 15 & $40.62 \AA^{2}$ \\
\hline
\end{tabular}

Note: ROF: Lipinski's Rule of Five; MW: Molecular weight $(\mathrm{g} / \mathrm{mol})$; $\mathrm{HBA}$ : Hydrogen bond acceptor; HBD: Hydrogen bond donor; Log P: Lipophilicity; nRB: Number of rotatable bond; TPSA: Topological polar surface area.

water molecule bridges at protein residues THR 296, SER 299, GLN 313 and ASN 427.

Markedly, licarin which does not show any interactions with SGTP4 protein residues in docking result showed significant hydrogen bonding interactions at residues ASN 34 and ILE 172 in MD (Figure 7c). Furthermore, the ligand showed hydrophobic interactions at protein residues LEU 35, TYR 176, ILE 295, LEU 302, and LEU 309 to VAL 316, while waterassisted interactions were seen in residues ASN 34, ILE 172, SER 175, ILE 295, and THR 296 to SER 299. As shown in Figure $8 \mathrm{c}$, harmonine showed significant hydrogen bonding between the ligand and SGTP4 protein at residues LEU 30, LEU 35, ASN 292, ASN 427, the hydrogen contact to residues ASN 34, GLU 38 and THR 296 are also shown by the docking results. The hydrophobic interaction of the ligand with SGTP4 protein is found in LEU 30, LEU 35, LEU 168 to TYR 176, ILE 295, and VAL 316 to PHE 391, while ASN 29, LEU 30, LEU 35, SER 73, ASN 292, ILE 295, GLN 313, LYS 315 and ASN 427 are the protein residues that formed water bridge interactions with SGTP4. More so, notable interactions for the water molecule bridges which are significant for the waterassisted ionic contacts between the ligand and the receptor are found in ASN 34, GLU 38 and THR 296, which corresponded with what was seen in the docking results.

\subsection{Oral bioavailability (ADME) analysis}

The failure of RO5 by a drug molecular compound does not depict its efficacy in treating certain diseases, but there is a high tendency of encountering an oral activity problem. And meeting the criteria of RO5 does not guarantee the druglikeness of the compound, because RO5 says nothing about the specific chemistry structural characteristics embedded in non-drugs or drug compounds (Lipinski, 2004). Although, $90 \%$ of orally active drug molecule which has bypassed the second phase of clinical trials, met the physicochemical parameter ranges of RO5 orally active molecular compound criteria, which are; $\log p \leq 5, \mathrm{MWT} \leq 500, \mathrm{H}$-bond acceptors $\leq$ 10, H-bond donors $\leq 5$ (Lipinski, 2004; Lipinski et al., 2001). These physicochemical parameters are usually associated with intestinal permeability and acceptable aqueous solubility as well as the steps one in oral bioavailability (Ojo et al., 2020). However, the RO5 of the molecular drug compounds used in this study was evaluated, and it was shown (Table 2) that licochalcone A, licarin, harmonine and praziquantel meet all the oral drugability and bioavailability of Lipinski's rule of five criteria. Praziquantel was predicted to meet all the criteria of the RO5 for an orally active drug molecule.

Subsequently, the Veber's rule which predicted the oral drugability of a molecular compound by meeting the following criteria; rotatable bond value less than or equal to 10 , hydrogen bond donors and acceptors less than or equal 12 , and the topological polar surface area not greater than 140 $\AA^{2}$ was further used to predict the oral bioavailability of the compounds. The Veber's rule also buttressed the results predicted by the RO5 that is, licochalcone A, licarin, harmonine and praziquantel; the control for orally active drug molecule. As aforementioned, RO5 does not say anything about the specific chemistry, structural properties of a compound and does not depict its efficacy in disease treatment, rather 
predict the oral bioavailability and intestinal permeability. Perhaps, if any compounds used in this study fails the oral bioavailability evaluation when the potency of the compound in treating schistosomiasis is finally ascertained experimentally, it could be delivered using a nanomedicine approach, which uses the application of nanotechnology (advanced drug delivery systems (DDS) and targeted approach). An approach used for monitoring, treatment, control and prevention of biological diseases and has been promising in improving pharmaceutical ingredients in the treatments of several diseases (Adekiya et al., 2020b). Alternatively, it could be administered using other routes of molecular active compound administration, such as intravenous, intramuscular among others.

\section{Conclusion}

Due to the challenges faced by PZQ, studies in the last few years have investigated various natural products from different chemical classes for their anti-schistosomal effect. In the current study, various computational approaches were employed to inhibit the activity of SGTP4; a druggable protein found on the surface of the schistosome tegument. The binding affinity and stability of the binding of four identified ligands against SGTP4 were similar to that of PZQ. Thus, providing a new avenue for schistosomiasis remedy by fasttracking the drug discovery process. Overall, further experimental validations are needed to have a deeper insight into how these ligands will elicit their inhibitory activities on SGTP4.

\section{Disclosure statement}

The authors declare that there are no conflict of interests.

\section{Author contributions}

TAA and RTA conceived the experiments. TAA, RTA, AK, AOF designed, performed, analysis the data and wrote the manuscript.

\section{ORCID}

Tayo A. Adekiya (D) 0000-0002-3382-9080

Raphael Taiwo Aruleba (D) http://orcid.org/0000-0003-0879-344X

Ashwil Klein (D) 0000-0002-5606-886X

Adewale O. Fadaka (D) 0000-0002-3952-2098

\section{References}

Adekiya, T. A., Aruleba, R. T., Khanyile, S., Masamba, P., Oyinloye, B. E., \& Kappo, A. P. (2017). Structural analysis and epitope prediction of MHC class-1-chain related protein-A for cancer vaccine development. Vaccines, 6(1), 1. https://doi.org/10.3390/vaccines6010001

Adekiya, T. A., Aruleba, R. T., Oyinloye, B. E., Okosun, K. O., \& Kappo, A. P. (2020a). The effect of climate change and the snail-schistosome cycle in transmission and bio-control of schistosomiasis in Sub-Saharan Africa. International Journal of Environmental Research and Public Health, 17(1), 181. https://doi.org/10.3390/ijerph17010181
Adekiya, T. A., Kappo, A. P., \& Okosun, K. O. (2017). Temperature and rainfall impact on schistosomiasis. Global Journal of Pure and Applied Mathematics, 13, 8453-8469.

Adekiya, T. A., Kondiah, P. P. D., Choonara, Y. E., Kumar, P., \& Pillay, V. (2020b). A review of nanotechnology for targeted anti-schistosomal therapy. Frontiers in Bioengineering and Biotechnology, 8, 32. https:// doi.org/10.3389/fbioe.2020.00032

Akabli, T., Lamchouri, F., Senhaji, S., \& Toufik, H. (2019). Molecular docking, ADME/Tox prediction, and in vitro study of the cell growth inhibitory activity of five $\beta$-carboline alkaloids. Structural Chemistry, 30(4), 1495-1504. https://doi.org/10.1007/s11224-019-01308-x

Aruleba, R. T., Adekiya, A. T., Molefe, P. F., Ikwegbue, P. C., Oyinloye, B. E., \& Kappo, A. P. (2020). Insights into functional amino acids of ULBP2 as potential immunogens against cancer. Scientific African, 10, e00581. https://doi.org/10.1016/j.sciaf.2020.e00581

Aruleba, R. T., Adekiya, T. A., Oyinloye, B. E., \& Kappo, A. P. (2018). Structural studies of predicted ligand binding sites and molecular docking analysis of Slc2a4 as a therapeutic target for the treatment of cancer. International Journal of Molecular Sciences, 19(2), 386. https://doi.org/10.3390/ijms19020386

Aruleba, R. T., Adekiya, T. A., Oyinloye, B. E., Masamba, P., Mbatha, L. S., Pretorius, A., \& Kappo, A. P. (2019). PZQ therapy: How close are we in the development of effective alternative anti-schistosomal drugs? Infectious Disorders Drug Targets, 19(4), 337-349. https://doi.org/10. 2174/1871526519666181231153139

Essmann, U., Perera, L., Berkowitz, M. L., Darden, T., Lee, H., \& Pedersen, L. G. (1995). A smooth particle mesh Ewald method. The Journal of Chemical Physics, 103(19), 8577-8593. https://doi.org/10.1063/1.470117

Fadaka, A. O., Bakare, O. O., Sibuyi, N. R. S., \& Klein, A. (2020). Gene expression alterations and molecular analysis of CHEK1 in solid tumors. Cancers, 12(3), 662. https://doi.org/10.3390/cancers12030662

Fadaka, A. O., Pretorius, A., \& Klein, A. (2019). MicroRNA assisted gene regulation in colorectal cancer. International Journal of Molecular Sciences, 20. https://doi.org/10.3390/ijms20194899

Hoover, W. G. (1985). Canonical dynamics: Equilibrium phase-space distributions. Physical Review. A, General Physics, 31(3), 1695-1697. https:// doi.org/10.1103/physreva.31.1695

Jorgensen, W. L., Chandrasekhar, J., Madura, J. D., Impey, R. W., \& Klein, M. L. (1983). Comparison of simple potential functions for simulating liquid water. The Journal of Chemical Physics, 79(2), 926-935. https:// doi.org/10.1063/1.445869

Kellershohn, J., Thomas, L., Hahnel, S. R., Grünweller, A., Hartmann, R. K., Hardt, M., Vilcinskas, A., Grevelding, C. G., \& Haeberlein, S. (2019). Insects in anthelminthics research: Lady beetle-derived harmonine affects survival, reproduction and stem cell proliferation of Schistosoma mansoni. PLoS Neglected Tropical Diseases, 13(3), e0007240. https://doi.org/10.1371/journal.pntd.0007240

Kim, S., Chen, J., Cheng, T., Gindulyte, A., He, J., He, S., Li, Q., Shoemaker, B. A., Thiessen, P. A., Yu, B., Zaslavsky, L., Zhang, J., \& Bolton, E. E. (2019). PubChem 2019 update: Improved access to chemical data. Nucleic Acids Research, 47(D1), D1102-D1109. https://doi.org/10.1093/ nar/gky1033

Krautz-Peterson, G., Simoes, M., Faghiri, Z., Ndegwa, D., Oliveira, G. Shoemaker, C. B., \& Skelly, P. J. (2010). Suppressing glucose transporter gene expression in schistosomes impairs parasite feeding and decreases survival in the mammalian host. PLoS Pathogens, 6(6), e1000932 https://doi.org/10.1371/journal.ppat.1000932

Laskowski, R. A., Rullmannn, J. A., MacArthur, M. W., Kaptein, R., \& Thornton, J. M. (1996). AQUA and PROCHECK-NMR: Programs for checking the quality of protein structures solved by NMR. Journal of Biomolecular NMR, 8(4), 477-486. https://doi.org/10.1007/BF00228148

Lipinski, C. A. (2004). Lead- and drug-like compounds: The rule-of-five revolution. Drug Discovery Today. Technologies, 1(4), 337-341. https:// doi.org/10.1016/j.ddtec.2004.11.007

Lipinski, C. A., Lombardo, F., Dominy, B. W., \& Feeney, P. J. (2001). Experimental and computational approaches to estimate solubility and permeability in drug discovery and development settings. Advanced Drug Delivery Reviews, 46(1-3), 3-26. DOI: 10.1016/s0169409x(00)00129-0 
Lyne, P. D., Lamb, M. L., \& Saeh, J. C. (2006). Accurate prediction of the relative potencies of members of a series of kinase inhibitors using molecular docking and MM-GBSA scoring. Journal of Medicinal Chemistry, 49(16), 4805-4808. https://doi.org/10.1021/jm060522a

MacDonald, K., Buxton, S., Kimber, M. J., Day, T. A., Robertson, A. P., \& Ribeiro, P. (2014). Functional characterization of a novel family of acetylcholine-gated chloride channels in Schistosoma mansoni. PLoS Pathogens, 10(6), e1004181. https://doi.org/10.1371/journal.ppat. 1004181

Mansour, T., \& Mansour, J. (2002). Targets in the tegument of flatworms. In Chemotherapeutic targets in parasites: Contemporary strategies (pp. 189-214). Cambridge University.

Meleti, V. R., Esperandim, V. R., Flauzino, L. G. B., Prizantelli, A. H., Paula, L. A. d L., Magalhães, L. G., Cunha, W. R., Laurentiz, R. d. S., Pissurno, A. P. d. R., Nanayakkara, N. P. D., Pereira, A. C., Bastos, J. K., Parreira, R. L. T., Orenha, R. P., \& E Silva, M. L. A. (2020). ( \pm )-Licarin A and its semi-synthetic derivatives: In vitro and in silico evaluation of trypanocidal and schistosomicidal activities. Acta Tropica, 202, 105248. https:// doi.org/10.1016/j.actatropica.2019.105248

Ojo, O. A., Afon, A. A., Ojo, A. B., Ajiboye, B. O., Okesola, M. A., Aruleba, R. T., Adekiya, T. A., \& Oyinloye, B. E. (2019). Spondias mombim L. (Anacardiaceae): Chemical fingerprints, inhibitory activities, and molecular docking on key enzymes relevant to erectile dysfunction and Alzheimer's diseases. Journal of Food Biochemistry, 43(3), e12772. https://doi.org/10.1111/jfbc. 12772

Ojo, O. A., Aruleba, R. T., Adekiya, T. A., Sibuyi, N. R. S., Ojo, A. B., Ajiboye, B. O., \& Oyinloye, B. E. (2020). Deciphering the interaction of puerarin with cancer macromolecules: An in silico investigation. Journal of Biomolecular Structure and Dynamics, 1-12. https://doi.org/ 10.1080/07391102.2020.1819425

Oyinloye, B. E., Adekiya, T. A., Aruleba, R. T., Ojo, O. A., \& Ajiboye, B. O. (2019). Structure-based docking studies of GLUT4 towards exploring selected phytochemicals from Solanum xanthocarpum as a therapeutic target for the treatment of cancer. Current Drug Discovery Technologies, 16(4), 406-416. https://doi.org/10.2174/1570163 815666180801152110

Pereira, A. C., Magalhães, L. G., Gonçalves, U. O., Luz, P. P., Moraes, A. C. G., Rodrigues, V., da Matta Guedes, P. M., da Silva Filho, A. A., Cunha, W. R., Bastos, J. K., Nanayakkara, N. P. D., \& e Silva, M. L. A.
(2011). Schistosomicidal and trypanocidal structure-activity relationships for ( \pm )-licarin A and its (-)- and (+)-enantiomers. Phytochemistry, 72(11-12), 1424-1430. https://doi.org/10.1016/j.phytochem.2011.04. 007

Skelly, P. J., \& Shoemaker, C. B. (1996). Rapid appearance and asymmetric distribution of glucose transporter SGTP4 at the apical surface of intramammalian-stage Schistosoma mansoni. Proceedings of the National Academy of Sciences of the United States of America, 93(8), 3642-3646. https://doi.org/10.1073/pnas.93.8.3642

Skelly, P. J., \& Shoemaker, C. B. (2001). The Schistosoma mansoni hostinteractive tegument forms from vesicle eruptions of a cyton network. Parasitology, 122 Pt 1(Pt 1), 67-73. https://doi.org/10.1017/ s0031182000007071

Skelly, P. J., Kim, J. W., Cunningham, J., \& Shoemaker, C. B. (1994). Cloning, characterization, and functional expression of cDNAs encoding glucose transporter proteins from the human parasite Schistosoma mansoni. The Journal of Biological Chemistry, 269(6), 4247-4253.

Skelly, P. J., Tielens, A. G., \& Shoemaker, C. B. (1998). Shoemaker CB glucose transport and metabolism in mammalian-stage schistosomes. Parasitology Today, 14(10), 402-406. https://doi.org/10.1016/s0169 4758(98)01319-2

Souza, R. L., Gonçalves, U. O., Badoco, F. R., de Souza Galvão, L., Santos, R. A. D., de Carvalho, P. H. D., de Carvalho, L. S. A., da Silva Filho, A. A., Veneziani, R. C. S., Rodrigues, V., Ambrósio, S. R., \& Magalhães, L. G. (2017). Licochalcone A induces morphological and biochemical alterations in Schistosoma mansoni adult worms. Biomedicine \& Pharmacotherapy=Biomedecine \& Pharmacotherapie, 96, 64-71. https://doi.org/10.1016/j.biopha.2017.09.128

Veber, D. F., Johnson, S. R., Cheng, H. Y., Smith, B. R., Ward, K. W., \& Kopple, K. D. (2002). Molecular properties that influence the oral bioavailability of drug candidates. Journal of Medicinal Chemistry, 45(12), 2615-2623. https://doi.org/10.1021/jm020017n

WHO. (2020). WHO fact sheets. https://www.who.int/health-topics/schistosomiasis\#tab=tab_1 (Retrived August 11, 2020)

Yadav, M., \& Khandelwal, S. (2019). Homology modeling and molecular dynamics dimulation study of $\beta$ carbonic anhydrase of Ascaris lumbricoides. Bioinformation, 15(8), 572-578. https://doi.org/10.6026/ 97320630015572 